\title{
Primary Yolk Sac Tumor of the Endometrium with MRI Findings: A
}

\section{Case Report}

\author{
Dalin $\mathrm{Zhu}^{1,{ }^{1,}, \text { Fan Feng }}{ }^{2}$ and Meijuan Peng ${ }^{1}$ \\ ${ }^{1}$ Department of Medical Imaging Center, Gansu Provincial Maternity and Child-Care Hospital, Lanzhou, China \\ ${ }^{2}$ Department of Reproductive Medicine Center, Gansu Provincial Maternity and Child-Care Hospital, Lanzhou, China \\ "Corresponding author: Department of Medical Imaging Center, Gansu Provincial Maternity and Child-Care Hosipital Lanzhou, Lanzhou, China. Tel: 86-9312605982, Email: \\ zdlldz@126.com
}

Received 2020 February 10; Revised 2020 June 06; Accepted 2020 June 13.

\begin{abstract}
Primary yolk sac tumor (YST) of the endometrium is a very rare malignant germ cell tumor, primary YST of the endometrium with detailed magnetic resonance imaging (MRI) and contrast-enhanced MRI (CE-MRI) findings have not yet been reported in the English literature. We report a 40-year-old woman presenting with irregular lower abdominal pain and dysmenorrhea for more than 5 months. MRI showed a lesion in the right side wall of the uterus. Postoperative pathology and immunohistochemistry confirmed the diagnosis of primary YST of the endometrium with the International Federation of Gynecology and Obstetrics (FIGO) system staging of IV. The signal of the tumor was complex and lacked specific characteristic. Diffusion-weighted imaging (DWI) showed that the lesion is diffuse limited, the average apparent diffusion coefficient (ADC) value of the solid component was $0.735 \times 10^{-3}$ $\mathrm{mm}^{2} / \mathrm{s}$. CE-MRI showed that the irregular lesions presented uneven and mild enhancement in the arterial stage, and continued to strengthen in the venous phase, and strengthened close to the myometrium in the delayed phase. Primary YST of the endometrium should be considered if MRI scans show an irregular lesion in the myometrium with high level of alpha-fetoprotein (AFP).
\end{abstract}

Keywords: Yolk Sac Tumor, Germ Cell Tumor, Magnetic Resonance Imaging

\section{Introduction}

Primary yolk sac tumor (YST) of the endometrium is very rare, and only 15 cases have been reported $(1,2)$. This case report is the 16 th case in the world. We first report the case of primary YST of the endometrium with detailed magnetic resonance imaging (MRI) and contrast enhanced (CE) MRI findings.

\section{Case Presentation}

The patient was a 40-year-old married woman with irregular lower abdominal pain and dysmenorrhea for more than 5 months. The patient felt that the lower abdominal pain was worse than before recently, accompanied by frequent urination. Tumor marker evaluations revealed a high level of AFP (1210 ng/mL), premenopausal Roman index (PI) (40.5) and human epididymis protein 4 (HE4) (121.2 pmol/mL). The levels of carbohydrate antigen (CA)125 (23.95 u/mL), CA 19 - 9 (11.11 U/mL), and carcinoembryonic antigen (CEA) $(1.48 \mathrm{ng} / \mathrm{mL})$ were normal.
Pelvic MRI and CE-MRI was performed using the Siemens Avanto $1.5 \mathrm{~T}$ system before surgery. MRI showed that the uterus was enlarged, the lesion located in the right side wall of the uterus, and the boundary was unclear, the serosal layer of the anterior wall of the uterus was unclear, and the tumor size was $7.7 \mathrm{~cm} \times 6.6 \mathrm{~cm}$ $\times 8.2 \mathrm{~cm}$. T1 weighted imaging (WI) showed the lesion is equal and slightly higher signal (Figure 1A). T2WI was equal, slightly lower and slightly higher signal (Figure 1B). Diffusion weighted imaging (DWI) indicated that the lesion was diffusion restricted (Figure $1 C$ ), and the average apparent diffusion coefficient (ADC) value of the solid component was $0.735 \times 10^{-3} \mathrm{~mm}^{2} / \mathrm{s}$, partially broke through the serosa layer, invaded the right side wall of the vagina and the paravaginal space, and the right uretera was dilated. CE-MRI showed that the lesions showed uneven and mild enhancement in the early stage (Figure 1D), and continued to strengthen in the venous phase (Figure $1 \mathrm{E}$ ), and strengthened close to the myometrium in the delayed phase (Figure $1 \mathrm{~F}$ ). No enlarged lymph nodes were present in the pelvis. 

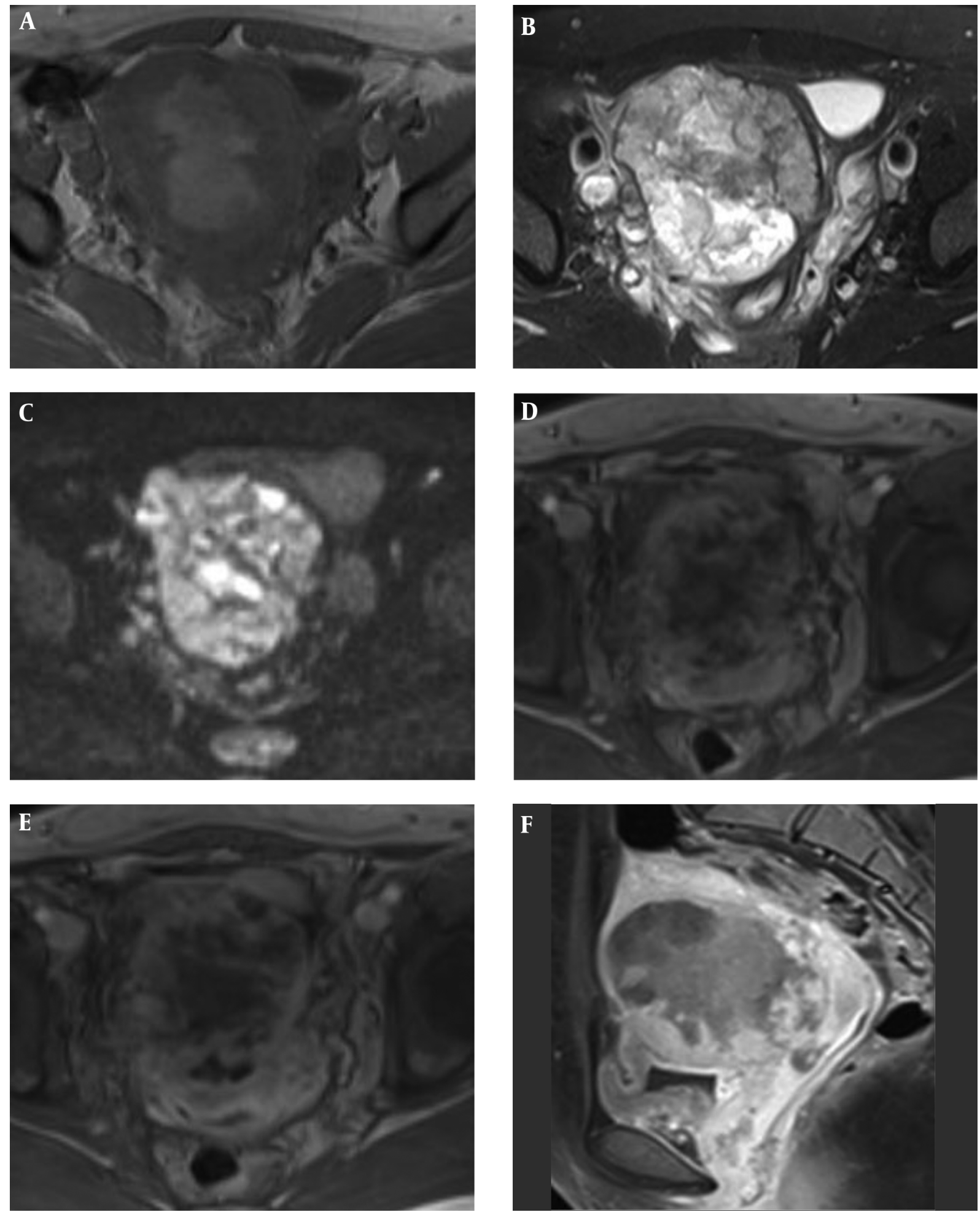

Figure 1. A 40-year-old woman with lower abdominal pain and dysmenorrhea who showed a lesion in the right side of the uterus in MRI. A, Axial T1WI imaging indicates that the lesion is iso- and slightly high-signal; B, Axial T2WI imaging indicates that the lesion is iso-, slightly low- and slightly high-signal; C, Axial DWI imaging indicates that the lesion shows diffusion restriction; D, Axial CE T1W MR imaging indicates that the lesion is uneven and shows mild enhancement in the early phase; E, Axial CE T1W MR imaging indicates that the lesion continues to strengthen in the venous phase; F, Delayed (3 minutes) contrast-enhanced sagittal T1WI shows the solid component of tumor enhanced close to the myometrium, multiple irregular unreinforced areas visible in the mass. 
The patient underwent an extensive uterus, and bilateral accessory resection, total cystectomy, and bilateral ureteral abdominal wall external surgery under general anesthesia. Postoperative pathological results showed: (1) tumor in the right side of the uterus, positive lymphatic invasion, positive vascular invasion, negative nerve invasion, positive bilateral uterine and its cutting edge invasion, positive vascular invasion of vaginal wall; (2) focal adenomyosis; (3) bladder invasion. (4) endometrium showed a proliferative phase reaction; (5) left ovary showed ovarian corpus luteum combined with bleeding; (6) right ovary showed ovarian inclusion cyst; (7) bilateral fallopian tubes showed no abnormalities. Serial AFP decreased to 2.42 ng/mL. Postoperative chemotherapy (cisplatin + vinblastine + bleomycin, PVB) chemotherapy for three courses. The patient is still alive after two years.

This case is in stage IV of FIGO (International Federation of Gynecology and Obstetrics). Postoperative pathological examination showed tumor in the right side of the uterus (Figure 2A). Tumor cells are dense and have an atypical network structure. Some tumor cells grow around the blood vessel wall, invade the surrounding muscular tissue, accompanied by a large amount of bleeding and necrosis (Figure 2B). The tumor cell nuclei are deeply stained, the nucleoli are obvious, and the nuclear membrane is irregular (Figure 2C).

\section{Discussion}

YST is a rare tumor in germ cell tumors. It most commonly occurs in the sacrococcygeal region, mediastinum, brain, vagina and retroperitoneum. Metastasis also occurs through the blood and lymphatic system. Primary YST of the endometrium is very rare (3). This case originated in the endometrium, was in stage IV of FIGO, and invaded the bladder and para-uterine structures.

T1WI and T2WI images of the pelvic MRI did not show specific findings, DWI image shows diffusion restricted area in the lesion. Hu et al. reported one case with MRI findings (4). The shape and signal of the tumor were similar to this case. They both show a place in the uterine muscle wall with complex signal, but no CE-MRI findings were reported in their case report. In this case, CE-MRI showed that the solid component of the mass appears as continued enhanced, the degree of enhance is close to the myometrium in the delayed phase.

Differential diagnoses for primary YST of the endometrium include endometrial cancer and uterine sarcoma. The MRI findings of endometrial cancer invading the muscular layer and primary YST of the endometrium is different. The early enhancement of CE-MR in endometrial cancer is lower than the normal endometrium and myometrium, and the enhancement signal of uterine myometrium is lower than myometrium in the delayed phase, which is helpful for differential diagnosis. The MRI findings of uterine sarcoma and primary YST of the endometrium is difficult to differentiate. MRI and CE-MRI findings are similar, but the high AFP level was showed in the primary YST of the endometrium.

In this case, MRI and CE-MRI imaging findings showed bilateral uterine, bladder and vaginal wall invasion, negative bilateral ovary and bilateral fallopian tube involvement is compatible with pathological assessments and extent of invasion determined after surgery. Typical pathology slice image of YST can be expressed as transparent body, papillary and gland-like structure, and the SchilerDuval (S-D) body is a typical feature $(5,6)$. The tumor cells are arranged in a single layer or a complex layer surrounding the blood vessel. A set of surrounding or endodermal sinus-like structures was formed around the blood vessels. This case was finally diagnosed as primary YST of the endometrium. YST can produce AFP. The measurement of AFP can be used for preoperative diagnosis and detection of metastasis and recurrence after treatment $(7,8)$. In this case, AFP increased significantly, reaching $1210 \mathrm{ng} / \mathrm{mL}$, and AFP dropped to $2.42 \mathrm{ng} / \mathrm{mL}$ after surgery. Review AFP was $1.43 \mathrm{ng} / \mathrm{mL}$ after 2 years. However, Song et al. reported a normal AFP level in a 38-year-old woman with primary YST of the endometrium (9). We need more sample collection and data for further research.

The clinical manifestation of primary YST of the endometrium is not specific. There are currently no uniform standards and regulations for the treatment of YST $(10,11)$. Fujita et al. (12) reported that 41 patients with ovarian endodermal sinus tumors underwent conservative surgery, followed by chemotherapy, with a 5-year and 10-year survival rate of approximately $80 \%$. Rossi et al. (13) reported a case of a 30-year-old woman who only had a uterus removed and retained bilateral attachments, and survived after 6 years. No tumor recurrence was seen after 2 years of follow-up. We will continue to follow up this case.

In summary, primary YST of the endometrium is a rarer malignant germ cell tumor with lack of specificity in clinical manifestations. Detection of AFP levels is important for diagnosis and postoperative follow-up, and the final diagnosis still requires pathology and immunohistochemistry. At present, there is no unified diagnosis and treatment guideline for primary YST of the endometrium. We 

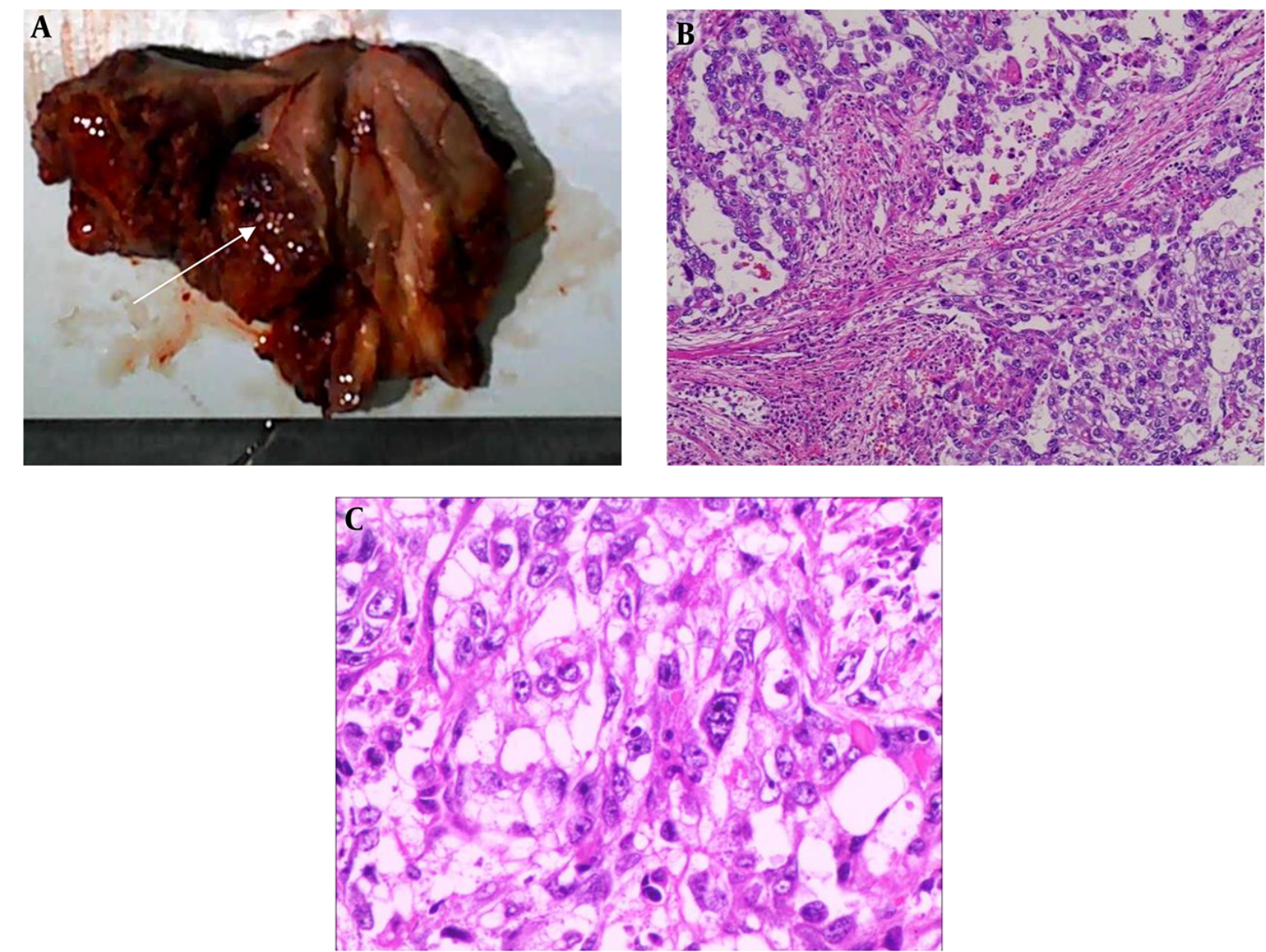

Figure 2. A, Photograph of the tumor (white arrow) following hysterectomy located in the right muscle wall of the uterus, tumor size was $6.5 \mathrm{~cm} \times 5 \mathrm{~cm} \times 3.5 \mathrm{~cm}$, and it showed tumor breaks through the uterine serosa; B, Images of primary yolk sac tumor (YST) of the endometrium. (H\&E stain, $\times 100$ ), Tumor cells are densely packed, partly enveloping blood vessels; C, Images of primary YST of the endometrium. (H\&E stain, $\times 400$ ), Tumor cells are deeply stained with nuclei, and nucleoli are obvious.

also believe that further researches and assessments are necessary.

\section{Footnotes}

Authors' Contribution: Dalin Zhu and Fan Feng made equal contribution to the article. Study concept and design: Dalin Zhu and Fan Feng. Drafting of the manuscript: Dalin Zhu and Meijuan Peng. Critical revision of the manuscript for important intellectual content: Dalin Zhu, Fan Feng, and Meijuan Peng.

Conflict of Interests: There are no conflicts of interest to declare.

Ethical Approval: Ethics Committee of Gansu provincial Maternity and Child-care Hospital approved this research (no.: 2018-16).
Funding/Support: This study was supported by Lanzhou Science and Technology Bureau of Gansu Province, project number (no.: 2018-3-30).

Informed Consent: Patient has provided informed consent for publication of the case.

\section{References}

1. Razzi S, Luisi S, Gabbanini M, Lazzeri L, Mazzini M, Petraglia F Yolk sac tumor in a young girl: A case report. Gynecol Endocrinol. 2005;20(6):334-5. doi: 10.1080/09513590500099065. [PubMed: 16019383].

2. Oguri H, Sumitomo R, Maeda N, Fukaya T, Moriki T. Primary yolk sac tumor concomitant with carcinosarcoma originating from the endometrium: Case report. Gynecol Oncol. 2006;103(1):368-71. doi: 10.1016/j.ygyno.2006.04.024. [PubMed: 16814851].

3. Zhang B, Gao S, Chen Y, Wu Y. Primary yolk sac tumor arising in the pancreas with hepatic metastasis: A case report. Korean J Radiol. 
2010;11(4):472-5. doi: 10.3348/kjr.2010.11.4.472. [PubMed: 20592932]. [PubMed Central: PMC2893319].

4. Hu Y, Zeng F, Xue M, Xiao S. A case report for primary yolk sac tumor of endometrium. J Cent South Univ Med Sci. 2016;41(12):1362-5.

5. Damato S, Haldar K, McCluggage WG. Primary endometrial yolk sac tumor with endodermal-intestinal differentiation masquerading as metastatic colorectal adenocarcinoma. Int J Gynecol Pathol. 2016;35(4):316-20. doi: 10.1097/PGP.0000000000000236. [PubMed: 26598980].

6. Abhilasha N, Bafna UD, Pallavi VR, Rathod PS, Krishnappa S. Primary yolk sac tumor of the endometrium: A rare entity. Indian J Cancer. 2014;51(4):446. doi: 10.4103/0019-509X.175315. [PubMed: 26842154].

7. Dede M, Pabuccu R, Yagci G, Yenen MC, Goktolga U, Gunhan O. Extragonadal yolk sac tumor in pelvic localization. A case report and literature review. Gynecol Oncol. 2004;92(3):989-91. doi: 10.1016/j.ygyno.2003.12.026. [PubMed: 14984973].

8. Qzler A, Dogan S, Mamedbeyli G, Rahatli S, Haberal AN, Dursun P, et al. Primary yolk sac tumor of endometrium: Report of two cases and review of literature. J Exp Ther Oncol. 2015;11(1):5-9. [PubMed: 26259383].

9. Song L, Wei X, Wang D, Yang K, Qie M, Yin R, et al. Primary yolk sac tumor originating from the endometrium: A case report and literature review. Medicine (Baltimore). 2019;98(15). e15144. doi: 10.1097/MD.0000000000015144. [PubMed: 30985686]. [PubMed Central: PMC6485813].

10. Nawa A, Obata N, Kikkawa F, Kawai M, Nagasaka T, Goto S, et al. Prognostic factors of patients with yolk sac tumors of the ovary. Am JObstet Gynecol. 2001;184(6):1182-8. doi: 10.1067/mob.2001.113323. [PubMed: 11349186].

11. Wang C, Li G, Xi L, Gu M, Ma D. Primary yolk sac tumor of the endometrium. Int J Gynaecol Obstet. 2011;114(3):291-3. doi: 10.1016/j.ijgo.2011.03.020. [PubMed: 21696729].

12. Fujita M, Inoue M, Tanizawa O, Minagawa J, Yamada T, Tani T. Retrospective review of 41 patients with endodermal sinus tumor of the ovary. Int J Gynecol Cancer. 1993;3(5):329-35. doi: 10.1046/j.15251438.1993.03050329.x. [PubMed: 11578366].

13. Rossi R, Stacchiotti D, Bernardini MG, Calvieri G, Lo Voi R. Primary yolk sac tumor of the endometrium: A case report and review of the literature. Am J Obstet Gynecol. 2011;204(4):e3-4. doi: 10.1016/j.ajog.2010.12.014. [PubMed: 21345404]. 